\title{
Molecular mechanism of LKB1 in the invasion and metastasis of colorectal cancer
}

\author{
YANGYANG CHEN $^{1,2^{*}}$, YIFEI LIU ${ }^{1 *}$, YANHONG ZHOU ${ }^{1 *}$ and HONGXIA YOU ${ }^{3}$ \\ ${ }^{1}$ Department of Gastroenterology, Hubei University of Science and Technology, Xianning, Hubei 437100; \\ ${ }^{2}$ Department of Pharmacy, The Second Affiliated Hospital of Southeast University, Nanjing, Jiangsu 210000; \\ ${ }^{3}$ Department of Gastroenterology, Wuhan University Affiliated Zhongshan Hospital, Wuhan, Hubei 430032, P.R. China
}

Received April 18, 2018; Accepted November 13, 2018

DOI: $10.3892 /$ or.2018.6877

\begin{abstract}
The occurrence of colorectal cancer (CRC) is associated with a variety of oncogenes and tumor-suppressor genes. As a tumor-suppressor gene, the liver kinase B1 gene (LKB1, also known as serine/threonine kinase 11, STK11) is closely related to tumor angiogenesis, invasion and metastasis, but its molecular mechanisms remain unclear. The aim of the present study was to investigate the effects of LKB1 on the invasion and metastasis of CRC, and to explore its molecular mechanisms. By detecting the expression of LKB1 in CRC, we can provide a reference index for diagnosing the depth of invasion and lymph node metastasis. Immunohistochemistry results indicated that LKB1 expression was strongly positive in normal colon tissue and that it inhibited the production of CRC. Immunocytochemical staining showed that the expression of LKB1 was significantly decreased in adenocarcinoma and mucinous adenocarcinoma tissues, and this reduced expression induced the invasion and metastasis of CRC. In the present study, LKB1 small interfering RNA (LKB1 siRNA) was transfected into LoVo cells to observe the effect of LKB1 on the invasion and metastasis of CRC. LKB1 silencing decreased the phosphorylation of AMP-activated protein kinase (p-AMPK) in its downstream pathway, which increased the phosphorylation of protein kinase B (p-AKT) and promoted tumor cell proliferation, enhancing the migration and invasion of CRC. The present study also explored the role of metformin in the LKB1 signaling pathway. Metformin inhibits the invasion and metastasis of CRC by activating p-AMPK, thereby inhibiting the activation of p-AKT. These results suggest that LKB1 plays an important role in the invasion and metastasis of CRC by activating AMPK, negatively regulating the AKT
\end{abstract}

Correspondence to: Professor Yanhong Zhou, Department of Gastroenterology, Hubei University of Science and Technology, 88 Xianning Avenue, Xianning, Hubei 437100, P.R. China

E-mail: yanhongzhou326@163.com

*Contributed equally

Key words: colorectal cancer, LKB1, invasion, metastasis signaling pathway and regulating gene expression. Mutation or deletion of LKB1 is expected to be a novel therapeutic target or clinical biomarker for the prevention of the invasion and metastasis of CRC.

\section{Introduction}

Colorectal cancer (CRC) is one of the most common malignant tumors in humans. When diagnosed with advanced metastatic disease, CRC patients traditionally have a poor prognosis, with 5 -year survival rates in the range of 5 to $8 \%(1,2)$. Clinical studies have shown that the main cause of death in patients with CRC is tumor invasion and metastasis to other tissues and organs (3). In addition to surgical resection of the primary tumor, the clinical key to improving patient survival and curing $\mathrm{CRC}$ is to inhibit angiogenesis, cutting off the transfer route and looking for new targets for effective drug treatment (4). The occurrence of CRC involves a variety of oncogenes and tumor-suppressor genes, although the underlying molecular mechanisms remain unclear $(5,6)$. The study of targeted gene therapy can provide a theoretical basis for the treatment of the invasion and metastasis of CRC.

As a tumor-suppressor gene, the liver kinase B1 gene (LKB1, also known as serine/threonine kinase 11, STK11) is closely related to tumor angiogenesis, invasion and metastasis $(7,8)$. The loss of LKB1 gene expression is related not only to the occurrence of a variety of tumors, such as CRC and thyroid carcinoma, but also to tumor angiogenesis and metastasis (9-11). Knockdown of LKB1 was found to enhance cell migration in human colon cancer HCT116 cells (12). The molecular mechanism by which the LKB1 pathway acts on angiogenesis and metastasis in CRC remains unclear. It is evident that small interfering RNAs (siRNAs) play an important role in cancer initiation and progression via the regulation of tumor-suppressor genes or oncogenes (13-15). In the present study, the LKB1 gene was silenced by siRNA, and the mechanism by which LKB1 affects the downstream invasion and metastasis of CRC cells was observed.

LKB1 has recently been identified as an upstream activating protein kinase of AMP-activated protein kinase (AMPK) (16). The study found that AMPK and the development of CRC are closely related $(17,18)$. Our studies revealed an important role played by AMPK alpha 1 (AMPKa1) in cell biology, connecting 
two hallmarks of tumor cells, namely, hyperproliferation and DNA damage, which may be due to reduction in the amount of p21 (19). These findings have important implications for understanding the molecular mechanisms by which AMPK acts as a promising tumor suppressor or senescence blocker. $\mathrm{Su}$ et al found that AMPK was closely related to angiogenesis in endothelial cells (20). LKB1 is a key regulator of the AMPK signaling pathway in some tissues, including liver, skeletal muscle, myocardium and various cancerous tissues $(21,22)$. Our data indicated that protein kinase B (AKT) is involved in the angiogenesis and metastasis of colon cancer. Aberrant AKT activation was found to promote the growth of a variety of LKB1-deficient tumors (23) by promoting the activation of vascular endothelial growth factor and angiogenic factors (24). Therefore, it is important to study the expression and regulation of LKB1 to clarify the mechanisms underlying the invasion and metastasis of CRC.

In the present study, immunohistochemistry (IHC) was used to detect the expression of the tumor-suppressor gene LKB1 in different colon tissues. CRC LoVo cells were transfected with LKB1 small interfering RNA (LKB1 siRNA). Metformin has the potential mechanism to inhibit tumor cell growth and reduce protein synthesis in CRC. The present study used LoVo cells treated with metformin to study the expression and biological significance of LKB1-AMPK-AKT to clarify the metastatic mechanism of CRC. We speculated that LKB1 activates AMPK, acting as a negative regulator of the AKT pathways and affecting the invasion and metastasis of colon cancer cells. In the present study, we explored the possibility of the prevention and treatment of the invasion and metastasis of colon cancer via the LKB1 pathway.

\section{Materials and methods}

Materials. The colon cancer tissue microarray was purchased from Fanpu Biotech, Inc. (cat. no. COC961; Guilin, China). The SP IHC kit and primary antibody dilution buffer were purchased from Sangon Biotech (Shanghai, China). The LoVo human CRC cell line was obtained from CHI Scientific (cat. no. CL-0144, Wuxi, China). The artificially synthesized LKB1 siRNA and RNase-free water were obtained from RiboBio (Guangzhou, China). Lipofectamine ${ }^{\mathrm{TM}} 2000$ was purchased from Thermo Fisher Scientific, Inc. (Waltham, MA, USA). All primary antibodies [LKB1 (dilution 1:1,000; cat. no. 3050S), p-LKB1 (dilution 1:1,000; cat. no. 3482S), AMPKa (dilution 1:1,000; cat. no. 2532S), p-AMPKa (Thr172, dilution 1:1,000; cat. no. 2535S), AKT (dilution 1:1,000; cat. no. 9272S), p-AKT (dilution 1:2,000; cat. no. 4060S)] were obtained from Cell Signaling Technology, Inc. (Danvers, MA, USA). The Prestained Dual Color Protein Molecular Weight Marker was purchased from Thermo Fisher Scientific, Inc. The Cell Counting Kit-8 (CCK-8) was purchased from Dojindo Laboratories, Co., Ltd. (Kyushu, Japan). Metformin was purchased from Sigma-Aldrich; Merck KGaA (Darmstadt, Germany). The study was approved by the Ethics Committee of Hubei University of Science and Technology (Xianning, China).

IHC. The rabbit anti-human LKB1 monoclonal antibody (dilution 1:200; cat. no. ab185734) was purchased from Sangon Biotech. The colorectal carcinoma chip was purchased from Fanpu Biotech, Inc. This chip included samples from 65 patients with different types of colorectal tumors and samples of normal and inflammatory polyps from 31 patients. The samples were adenocarcinoma and mucinous adenocarcinoma tissues from 65 patients. Of the 65 patients, 35 were male and 30 were female; 36 were younger than 60 years, while 29 were older than 60 years. The TNM staging of colon cancer was carried out according to the 8th edition of the AJCC Cancer Staging Manual (25). In the present study, we observed the effect of LKB1 on metastasis of CRC. Therefore, the lymph node metastasis status in the present study was divided into two types: No metastasis and metastasis, and no detailed distinction was made. The 65 colorectal tumor samples were composed of 20 cases of adenocarcinoma with pathological grade I, 31 cases of adenocarcinoma with pathological grade II, and 14 cases of adenocarcinoma with pathological grade III. The tissues were cut into $4-\mu \mathrm{m}$-thick slices. The IHC SP method was performed according to the manufacturer's instructions, with known positive tissue sections as a positive control and phosphate-buffered saline (PBS) instead of primary antibody as a negative control. The procedure was conducted as follows: i) Dewaxing and hydration with a graded ethanol series moving from a high ethanol concentration to a low ethanol concentration; ii) antigen repair; iii) incubation with normal goat serum solution at $37^{\circ} \mathrm{C}$ for $10 \mathrm{~min}$; iv) application of the primary antibody LKB1; dilution $1: 200$ ) at $4^{\circ} \mathrm{C}$ and incubation overnight, washing with PBS 3 times for $3 \mathrm{~min}$ each time, incubation with the mouse anti-rabbit secondary antibody (dilution 1:200; cat. no. BM2004; Wuhan Boster Biological Technology Co., Ltd., Wuhan, China) at $37^{\circ} \mathrm{C}$ for $1 \mathrm{~h}$ and washing with PBS 3 times for 3 min each time; v) application of the horseradish peroxidase-labeled streptomycin-avidin working solution and washing with PBS 3 times for 3 min each time; vi) $\mathrm{DAB} / \mathrm{H}_{2} \mathrm{O}_{2}$ reactive dyeing followed by thorough rinsing with water, hematoxylin staining, dehydration, transparent and drying; vii) mounting with a neutral gum with no bubbles. The sections were scanned at magnification (x200) using an light microscopy (Olympus Corp. Tokyo, Japan). The intensity of the staining as well as the percentage of positive cells was recorded. Staining intensity was scored from 0 to $3+$; the intensity score was established as follows: 0 if tumor cells had complete absence of staining or faint staining intensity in $<10 \%$; $1+$ if $>10 \%$ of tumor cells had faint staining; $2+$ if tumor cells had moderate staining; and $3+$ if tumor cells had strong staining. Tumors with $1+, 2+$ and $3+$ expression were interpreted as positive for LKB1 antibody expression, and tumors with no expression (0 score) were interpreted as negative.

Cell culture. LoVo cells were maintained in RPMI-1640 culture medium supplemented with $10 \%$ fetal bovine serum (FBS; CHI Scientific) with $1 \%$ penicillin-streptomycin (HyClone; GE Healthcare Life Sciences, Logan, UT, USA). The cells were grown in monolayer cultures with $5 \% \mathrm{CO}_{2}$ in a humidified $37^{\circ} \mathrm{C}$ incubator. Every 2-3 days, the cells were subcultured. When the cells reached the logarithmic growth phase, $0.25 \%$ trypsin (Gibco Invitrogen; Thermo Fisher Scientific, Inc.) was applied for 1-3 min. The cells were resuspended in RPMI-1640 containing $10 \% \mathrm{FBS}$ at a cell concentration of $1 \times 10^{4}$ cells $/ \mathrm{ml}$. 
The experimental groups were as follows: i) blank control group, untransfected LoVo cells; ii) metformin group, LoVo cells treated with $20 \mathrm{mmol} / 1$ metformin; iii) LKB1 siRNA group, LoVo cells transfected with LKB1 siRNA; and iv) LKB1 siRNA+metformin group, LoVo cells transfected with LKB1 siRNA were also treated with $20 \mathrm{mmol} / 1$ metformin.

LKB1 siRNA synthesis and transfection. The cells were divided into the following 3 groups: i) blank control group, untransfected LoVo cells; ii) negative control group, LoVo cells transfected with Lipofectamine $^{\mathrm{TM}}$ 2000; iii) positive control group (LKB1 siRNA group), transfected with LKB1 siRNA. Appropriate positive and negative controls were run simultaneously. In total, 3 groups of cells were transfected with siRNAs the sequences of which were specific for LKB1 (namely, siRNA-001, siRNA-002 and siRNA-003). Another group of cells was transfected with a fluorescein amidite (FAM)-labeled non-specific siRNA that served as the negative control (NC) siRNA. The sequences of the siRNAs used in the present study were as follows: siRNA-001 sense, 5'-GAAGAAGGAAAUUCAACU A-3' and antisense, 5'-UAGUUGAAUUUCCUUCUUC-3'; siRNA-002sense, 5'-GCUGGUUCCGGAAGAAACA-3' and antisense, 5'-UGUUUCUUCCGGAACCAGC-3'; siRNA-003 sense, 5'-GGACUGACGUGUAGAACAA-3' and antisense, 5'-UUGUUCUACACGUCAGUCC-3'. In the present study, the risk of this potential off-target effect of siRNA affected the application of siRNA in this technology gene therapy field. LKB1 siRNA was synthesized by Guangzhou RiboBio Co., Ltd. The company is careful to avoid the off-target effect when designing siRNA, and the company offers siRNA modification. The company conducts product quality testing after the product is produced. For cell transfection, the cells were plated on 6-well plates ( $2 \times 10^{5}$ cells for LoVo) in RPMI-1640 with $10 \%$ FBS. After $12 \mathrm{~h}, 10 \mu \mathrm{l}$ of siRNA/well was incubated with $100 \mu \mathrm{l}$ of Opti-MEM, and $5 \mu \mathrm{l}$ of Lipofectamine ${ }^{\mathrm{TM}} 2000$ was diluted with $100 \mu \mathrm{l}$ of Opti-MEM; $200 \mu \mathrm{l}$ of the above mixture was added/well according to the manufacturer's instructions. The transfected cells were incubated in a humidified $37^{\circ} \mathrm{C}$ incubator with $5 \% \mathrm{CO}_{2}$ for $48 \mathrm{~h}$.

Examination of morphological changes. Following the application of LoVo-Con siRNA or LKB1 siRNA for $48 \mathrm{~h}$, an inverted phase-contrast microscope (Nikon Corporation, Tokyo, Japan) was utilized to observe the morphological changes in the cells. Images were captured using a digital camera (Nikon Corporation) at a magnification of x200.

$C C K-8$ assay. The effect on cell proliferation and toxicity due to metformin were assessed using a CCK-8 assay. Briefly, $3 \times 10^{4}$ cells/well were seeded in 96-well culture plates. After overnight incubation, the cells were treated with varying concentrations of metformin for 24,48 and $72 \mathrm{~h}$. The concentrations of metformin were $0,5,10,15,20$ and $25 \mathrm{mmol} / \mathrm{l}$. A total of $20 \mu \mathrm{l}$ of CCK-8 working solution was added to each well, followed by incubation for $4 \mathrm{~h}$ at $37^{\circ} \mathrm{C}$, and the absorbance was finally measured at $450 \mathrm{~nm}$ using a Model 3550 Microplate Reader (Bio-Rad Laboratories, Inc., Hercules, CA, USA).

Migration and invasion assays. Transwell chambers (Corning, Inc., Corning, NY, USA) were used to perform the cell migration and invasion assays. The cells were inoculated into 6-well plate with RPMI-1640 containing 10\% FBS at a cell concentration of $2 \times 10^{4}$ cells $/ \mathrm{ml}$. First, $800 \mu \mathrm{l}$ of RPMI-1640 medium plus $10 \%$ FBS was added to a 24-well plate, and then the plate was inserted into the Transwell chamber. After $1 \mathrm{~h}, 200 \mu \mathrm{l}$ of cell suspension was added to the upper Transwell chamber. The cells were grown in monolayer cultures with $5 \% \mathrm{CO}_{2}$ in a humidified $37^{\circ} \mathrm{C}$ incubator. After $24 \mathrm{~h}$, the Transwell chamber was washed once with sterile PBS. The cells that had not migrated were wiped off the upper surface with a clean cotton ball, and then a $10 \%$ methanol solution was used to fix the migrated cells for $30 \mathrm{~min}$. The film was carefully cut and a drop of 5\% crystal violet dye was added to the membrane. The cells were incubated at room temperature for $20 \mathrm{~min}$, and then washed once with PBS. The slide was fixed with neutral gum. Finally, the purple-blue cells were observed and counted under the multifield view of the microscope. The number of tumor cells was calculated in 5 random fields at a magnification of $\mathrm{x} 200$, using an inverted microscope (Olympus DP7; Olympus Corp.) and expressed as the average number of cells/field of view. The number of migrating cells present in 9 random fields was counted, and the experiment was repeated $\geq 3$ times. Invasion assays were performed using the same protocol, except with Matrigel (BD Biosciences, San Jose, CA, USA)-coated Transwell chambers and incubation for $24 \mathrm{~h}$ at $37^{\circ} \mathrm{C}$.

Western blot analysis. Protein lysates from cells were extracted in ice-cold lysis buffer [10 $\mu \mathrm{l}$ PMSF, $10 \mu 1 \mathrm{NaVO}_{3}, 10 \mu \mathrm{l}$ phosphatase inhibitor cocktail and $10 \mu \mathrm{l}$ protease inhibitor cocktail (Sigma-Aldrich; Merck KGaA) in $1 \mathrm{ml}$ RIPA]. Total protein was subjected to SDS-PAGE followed by immunoblotting. The total protein content was quantitated according to the Bradford method. Purified protein lysates $(40 \mu \mathrm{g})$ were separated electrophoretically on $12 \%$ denaturing SDS polyacrylamide gels (separating gel) and 5\% denaturing SDS polyacrylamide gel (concentrated gum). Then, the protein was transferred to nitrocellulose membranes. After blocking with 5\% non-fat dry milk, the membranes were probed with primary antibodies [LKB1 (dilution 1:1,000); p-LKB1 (dilution 1:1,000); AMPKa (dilution 1:1,000); p-AMPKa (Thr172, dilution 1:1,000); AKT (dilution 1:1,000); p-AKT (dilution 1:2,000); $\beta$-actin mouse monoclonal antibody (dilution 1:2,000; cat. no. BM0627; Wuhan Boster Biological Technology, Co., Ltd.)] at $4^{\circ} \mathrm{C}$ and incubation overnight. The immunoblotted proteins were visualized using the goat anti-mouse secondary antibody (dilution 1:5,000; cat. no. BA1001; Wuhan Boster Biological Technology, Co., Ltd.) or mouse anti-rabbit secondary antibody (dilution 1:5,000; cat. no. BM2004; Wuhan Boster Biological Technology Co., Ltd.) and detected using enhanced chemiluminescence (ECL) substrate (Cell Signaling Technology, Inc.). Appropriate positive and negative controls were run simultaneously. Western blot analysis was repeated 2-4 times. The protein bands were analyzed and quantified using a Bio-Profil Image Analysis system (Vilber Lourmat, Marne La Vallee, France), and the protein expression levels were expressed in relative units (RU). The results were normalized to actin.

Statistical analysis. The statistical software package SPSS 21.0 (IBM Corp., Armonk, NY, USA) was used for the statistical analysis. The experimental results are presented as the mean \pm standard deviation (mean $\pm \mathrm{SD})$. The data 
Table I. Immunohistochemical staining of LKB1 in CRC and normal colon mucosa.

\begin{tabular}{lcccc}
\hline Groups & Total & $\begin{array}{c}\text { Strong positive } \\
\text { staining n }(\%)\end{array}$ & $\begin{array}{c}\text { Positive } \\
\text { staining n }(\%)\end{array}$ & $\begin{array}{c}\text { Negative } \\
\text { staining n }(\%)\end{array}$ \\
\hline Normal tissue & 10 & $10(100.0)$ & $0(0)$ & $0(0)$ \\
Inflammatory polyp & 21 & $0(0)$ & $12(57.1)$ & $9(42.9)$ \\
Adenocarcinoma/mucinous adenocarcinoma & 65 & $0(0)$ & $28(43.1)$ & $37(56.9)$ \\
\hline
\end{tabular}

LKB1, liver kinase B1 gene; CRC, colorectal cancer.

Table II. Association between clinicopathological factors and LKB1 protein expression as determined by IHC.

\begin{tabular}{|c|c|c|c|c|c|}
\hline \multirow[b]{2}{*}{ Parameter } & \multirow[b]{2}{*}{ Cases } & \multicolumn{2}{|c|}{ LKB1 } & \multirow[b]{2}{*}{$\chi^{2}$} & \multirow[b]{2}{*}{ P-value } \\
\hline & & Positive & Negative & & \\
\hline \multicolumn{6}{|l|}{ Sex } \\
\hline Male & 35 & 19 & 16 & \multirow[t]{2}{*}{0.215} & \multirow[t]{2}{*}{0.643} \\
\hline Female & 30 & 18 & 12 & & \\
\hline \multicolumn{6}{|c|}{ Age (years) } \\
\hline$\leq 60$ & 36 & 22 & 14 & \multirow[t]{2}{*}{0.041} & \multirow[t]{2}{*}{0.839} \\
\hline$>60$ & 29 & 17 & 12 & & \\
\hline \multicolumn{6}{|c|}{ Degree of differentiation } \\
\hline $\mathrm{I}$ & 20 & 15 & 5 & \multirow[t]{3}{*}{2.550} & \multirow[t]{3}{*}{0.279} \\
\hline II & 31 & 18 & 13 & & \\
\hline III & 14 & 7 & 7 & & \\
\hline \multicolumn{6}{|c|}{ Infiltrating depth } \\
\hline $\mathrm{T} 1+\mathrm{T} 2$ & 35 & 17 & 18 & \multirow[t]{2}{*}{4.205} & \multirow[t]{2}{*}{0.004} \\
\hline T3 & 30 & 22 & 8 & & \\
\hline \multicolumn{6}{|c|}{ Lymph node m]etastasis } \\
\hline No & 37 & 15 & 22 & \multirow[t]{2}{*}{9.802} & \multirow[t]{2}{*}{0.002} \\
\hline Yes & 28 & 22 & 6 & & \\
\hline
\end{tabular}

LKB1, liver kinase B1 gene; IHC, immunohistochemistry.

analysis was performed with one-way ANOVA and $\chi^{2}$ tests. One-way analysis of variance with least significant difference (LSD) post hoc test for multiple comparisons was performed. P-value $<0.05$ was considered to indicate a statistically significant result.

\section{Results}

Expression of LKBI protein in CRC and normal colon mucosa. LKB1 protein expression was evaluated using IHC staining in 96 cases. LKB1 protein expression and staining scores were observed in the cytoplasm and the nucleus. The expression of LKB1 was detected in the cytoplasm and nuclei of normal colon tissue, and positive expression was observed in 10 cases $(100 \%)$. Positive expression of LKB1 was identified in $12(57.1 \%)$ of the inflammatory polyp tissue samples examined, and negative expression was identified in 9 cases (42.9\%). LKB1 expression was detected in the cytoplasm and nucleus of the CRC cells, and positive expression was observed in 28 cases $(43.1 \%)$, while negative expression was detected in 37 cases (56.9\%). LKB1 expression in different tissues was significantly different $(\mathrm{P}<0.01$; Table I and Fig. 1).

The association between LKB1 expression and clinicopathological factors was analyzed in patients with CRC (Table II). The positive expression of LKB1 was significantly associated with the depth of invasion and lymph node metastasis $\left(\chi^{2}=4.205\right.$ and 9.802; $\left.\mathrm{P}<0.01\right)$. However, LKB1 expression was not associated with sex, age or the degree of differentiation $\left(\chi^{2}=0.215,0.041\right.$ and 2.550; $\left.P>0.05\right)$.

Effect of LKB1 siRNA on the morphology of LoVo cells. After transfection, the morphological changes in the different groups were observed by inverted microscope. There was no significant difference between the negative control group and the blank control group. The apoptotic cells in the present study refer to cells that can be seen to float in the culture solution. Compared with the negative control group, cell proliferation was increased and the number of cells was increased in the LKB1 siRNA group, but there was a small number of apoptotic cells, indicating toxicity to some cells (Fig. 2). 

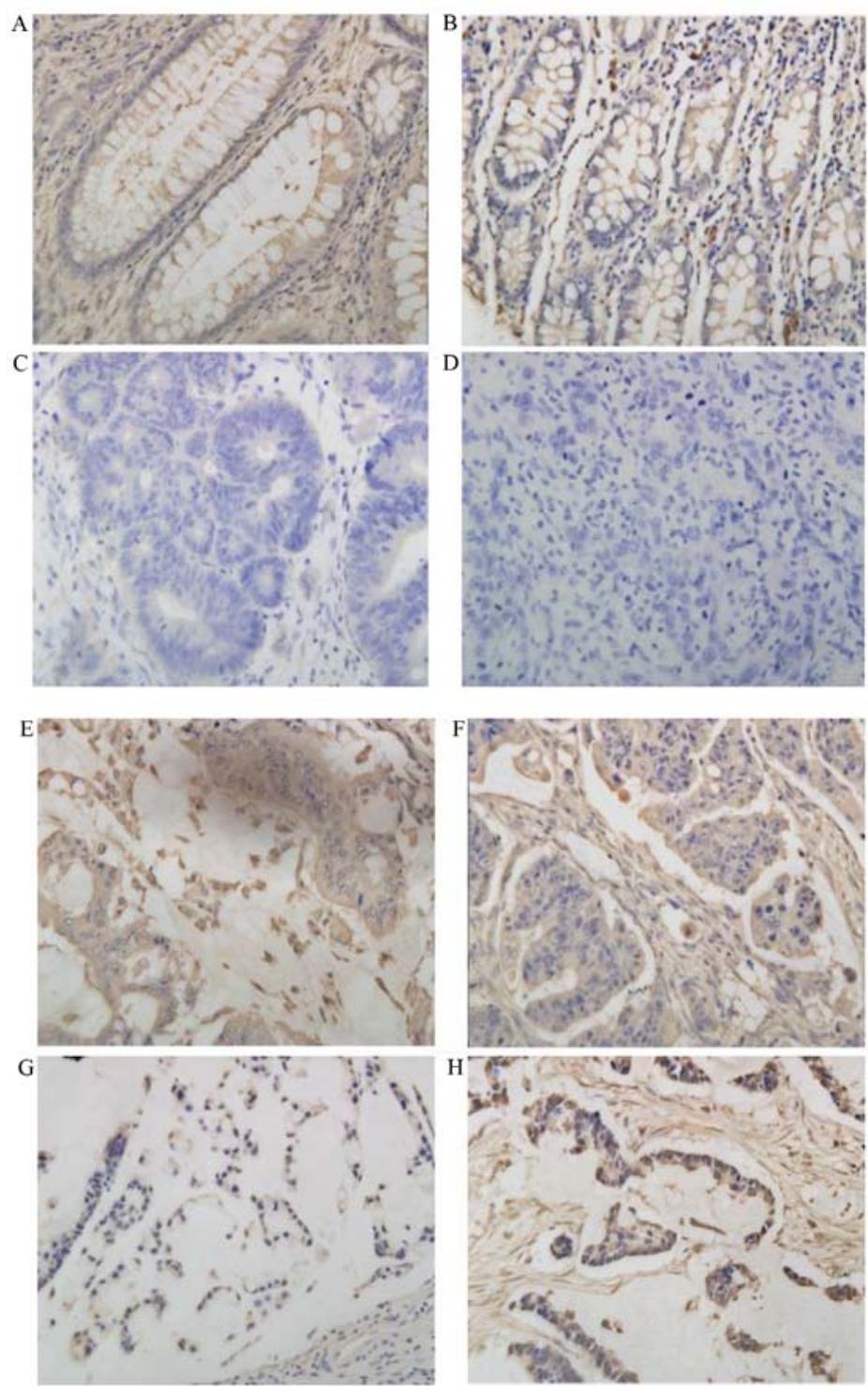

Figure 1. Immunohistochemical staining of LKB1 in colorectal carcinoma tissue sections (magnification, x200). (A and B) Normal tissue (positive). (C and D) Adenocarcinoma (negative). (E and F) Adenocarcinoma (positive). (G and H) Adenocarcinoma: Mucinous adenocarcinoma (positive). LKB1, liver kinase B1 gene.
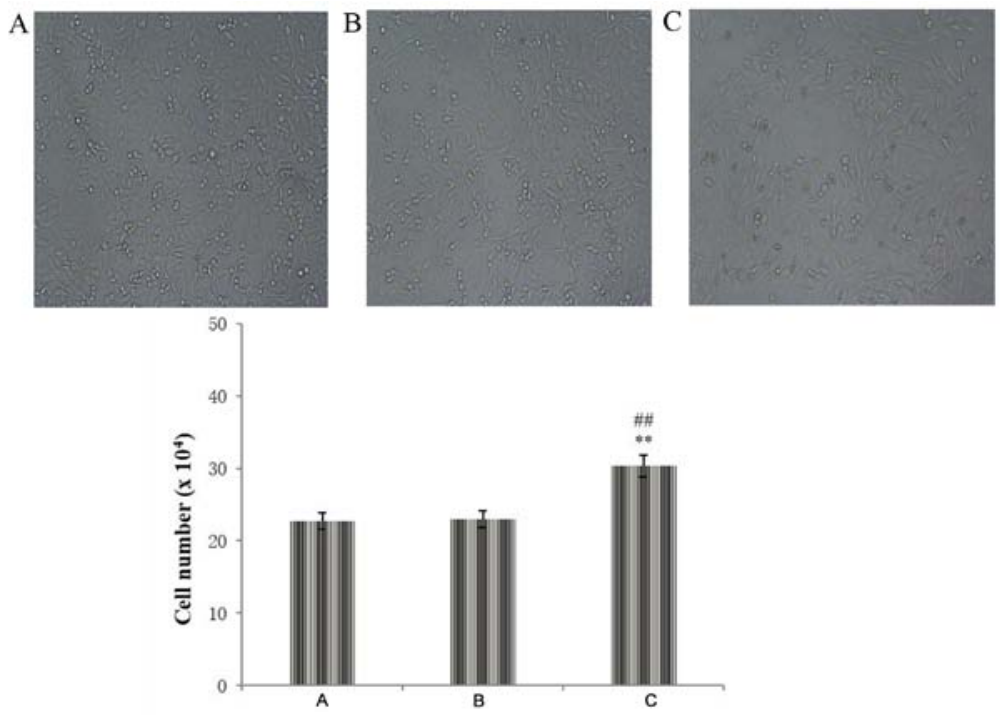

Figure 2. Effect of LKB1 siRNA on the morphological changes of LoVo cells magnification, x200). (A) Blank control group. (B) Negative control group. (C) LKB1 siRNA group. ${ }^{* *} \mathrm{P}<0.01$ vs. blank control group; ${ }^{\# \#} \mathrm{P}<0.01$ vs. negative control group. LKB1, liver kinase $\mathrm{B} 1$ gene. 

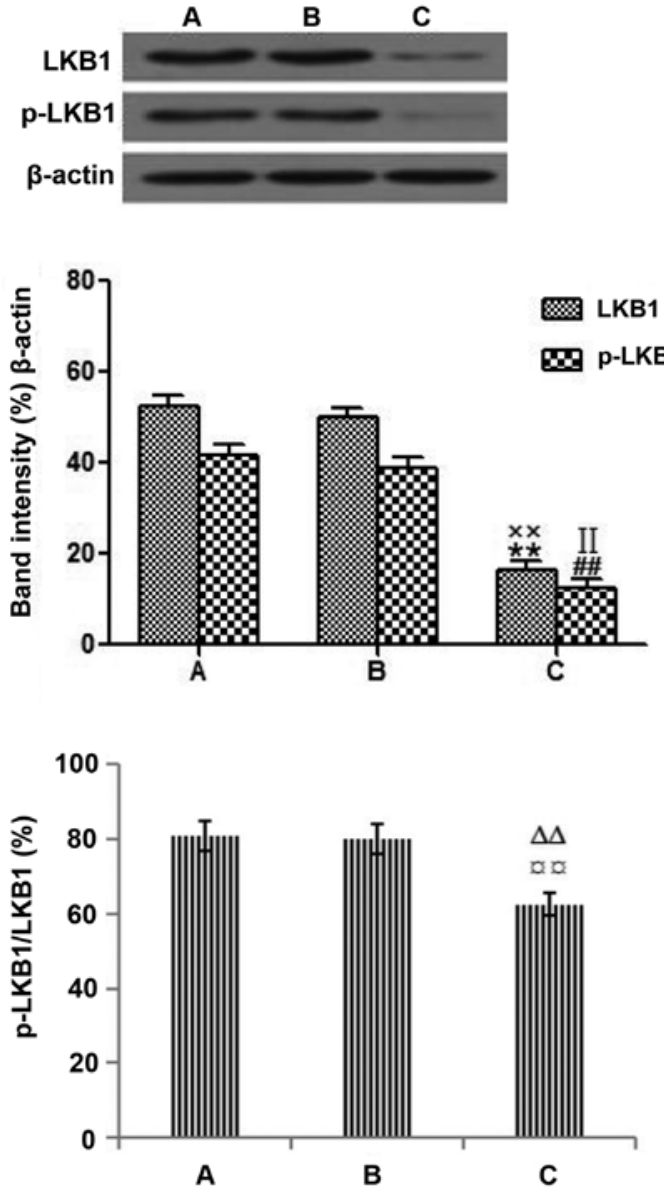

Figure 3. Western blot detection of LKB1 protein expression. (A) Blank control group. (B) Negative control group. (C) LKB1 siRNA group. ${ }^{* *} \mathrm{P}<0.01$ (LKB1), ${ }^{\# \#} \mathrm{P}<0.01$ (p-LKB1) vs. blank control group; ${ }^{\mathrm{x}} \mathrm{P}<0.01$ (LKB1), ${ }^{\mathrm{II}} \mathrm{P}<0.01$ (p-LKB1) vs. negative control group. ${ }^{\Delta \Delta} \mathrm{P}<0.01$ (p-LKB1/LKB1) vs. blank control group; ${ }^{a \circ} \mathrm{P}<0.01$ (p-LKB1/LKB1) vs. negative control group. LKB1, liver kinase B1 gene; $\mathrm{p}$, phosphorylated.

Western blotting detection of transfected cells. Following transfection of the LoVo cells with LKB1 siRNA for $48 \mathrm{~h}$, western blotting was performed. LKB1 siRNA was synthesized by Guangzhou RiboBio Co., Ltd. The company tested that siRNA 001 had the best transfection efficiency. According to the company's synthetic results, the present study used siRNA-001 (data not shown). The expression levels of LKB1 and p-LKB1 in the LKB1 siRNA group were markedly decreased compared with those in the blank control group. Results indicate that $\mathrm{p}-\mathrm{LKB} 1 / \mathrm{LKB} 1$ expression levels were lower in the LKB1 siRNA group, compare to the negative control group (Fig. 3). The results confirmed that LKB1 siRNA had been successfully transfected into the LoVo cells.

Effect of metformin on cell proliferation as detected by $C C K-8$ assay. Metformin significantly inhibited the proliferation of LoVo cells in a dose- and time-dependent manner. The $\mathrm{IC}_{50}$ of metformin was $20 \mathrm{mmol} / \mathrm{l}$ after $48 \mathrm{~h}$ of treatment (Fig. 4, Table III).

Effect ofmetformin on the morphology of LoVocells. Compared with the blank control group, treatment with metformin resulted in apoptotic morphological changes in LoVo cells.

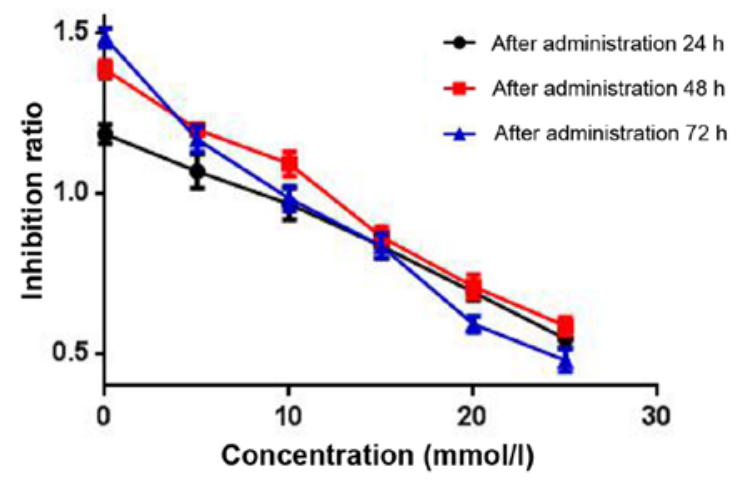

Figure 4. Effect of metformin on LoVo cell proliferation at 24, 48 and $72 \mathrm{~h}$. Data are presented as the mean $\pm \operatorname{SD}(n=6)$.
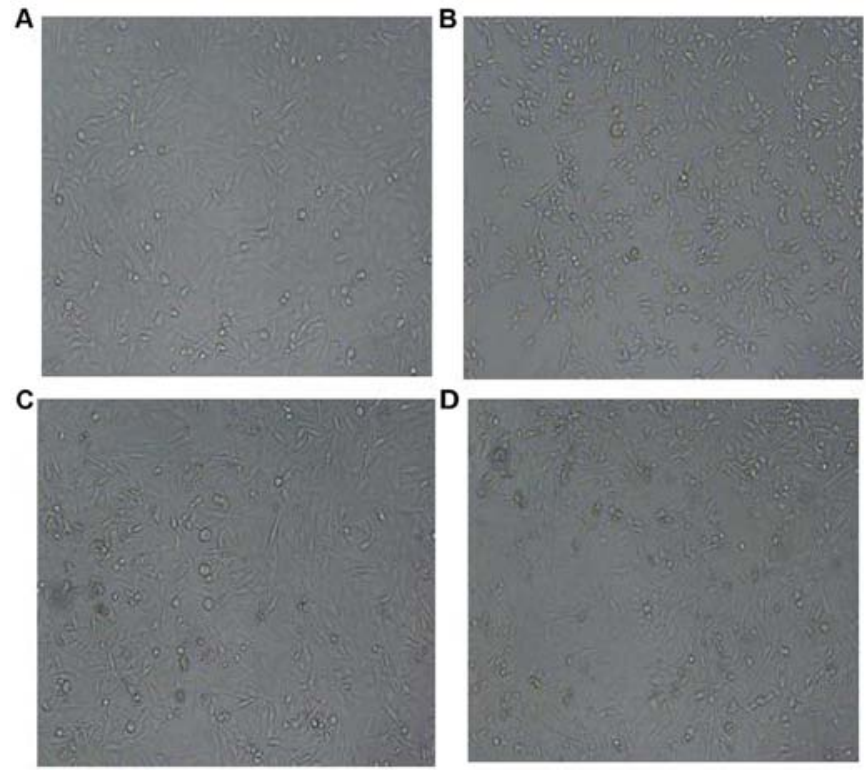

Figure 5. Effect of metformin on the morphology of LoVo cells (magnification, $\mathrm{x} 200$ ). (A) Blank control group. (B) Metformin group. (C) LKB1 siRNA group. (D) LKB1 siRNA+metformin group. LKB1, liver kinase B1 gene.

Under the light microscope, we observed that the shape of apoptotic cells became smaller, rounded and the adherent cells shrank and desquamated. The LKB1 siRNA group indicated that deletion of LKB1 led to cell proliferation (Fig. 5).

Effects of LKB1 on the migration and invasion of LoVo cells. A Transwell chamber assay was used to determine the migration and invasion abilities of LoVo cells. As shown in Figs. 6 and 7, the migration and invasion capabilities were clearly increased in the LKB1 siRNA group compared with the blank control group $(\mathrm{P}<0.01)$. The results demonstrated that the deletion of LKB1 increased the migration and invasion capacities of CRC cells. The results of the Transwell chamber experiments in the study indicated that the migration and invasion abilities of the CRC cells with deletion of LKB1 were enhanced, which indicated that the metastasis of CRC was enhanced.

To further explore whether the LKB1 signaling pathway is associated with the migration and invasion of LoVo cells, LoVo cells were treated with $20 \mathrm{mmol} / 1$ metformin for 48 h. As shown in Figs. 6 and 7, the migration and invasion 
Table III. Inhibitory rate of cell growth of LoVo cells treated with metformin by CCK-8 assay.

\begin{tabular}{lccc}
\hline Group & $24 \mathrm{~h}$ & $48 \mathrm{~h}$ & $72 \mathrm{~h}$ \\
\hline Blank control group & $0.000+0.000$ & $0.00+0.00$ & $0.00+0.00$ \\
Negative control group & $1.186+0.029$ & $1.388+0.027$ & $1.486+0.029$ \\
Metformin group (mmol/l) & & \\
5 & $1.070+0.052^{\mathrm{a}}$ & $1.199+0.099^{\mathrm{a}, \mathrm{b}}$ & $1.169+0.040^{\mathrm{a}, \mathrm{b}}$ \\
10 & $0.967+0.049^{\mathrm{a}, \mathrm{c}}$ & $1.093+0.038^{\mathrm{a}-\mathrm{c}}$ & $0.984+0.038^{\mathrm{a}-\mathrm{c}}$ \\
15 & $0.084+0.038^{\mathrm{a}, \mathrm{c}}$ & $0.086+0.031^{\mathrm{a}-\mathrm{c}}$ & $0.084+0.038^{\mathrm{a}-\mathrm{c}}$ \\
20 & $0.069+0.025^{\mathrm{a}, \mathrm{c}}$ & $0.513+0.037^{\mathrm{a}-\mathrm{c}}$ & $0.710+0.025^{\mathrm{a}-\mathrm{c}}$ \\
25 & $0.547+0.027^{\mathrm{a}, \mathrm{c}}$ & $0.587+0.028^{\mathrm{a}-\mathrm{c}}$ & $0.480+0.035^{\mathrm{a}-\mathrm{c}}$
\end{tabular}

Metformin group vs. negative control group $\left({ }^{a} \mathrm{P}<0.05\right)$. Difference in terms of time was statistically significant in the same concentration group $\left({ }^{\mathrm{b}} \mathrm{P}<0.05\right)$. Difference in terms of concentration was statistically significant in the same time group $\left({ }^{\mathrm{c}} \mathrm{P}<0.05\right)$.
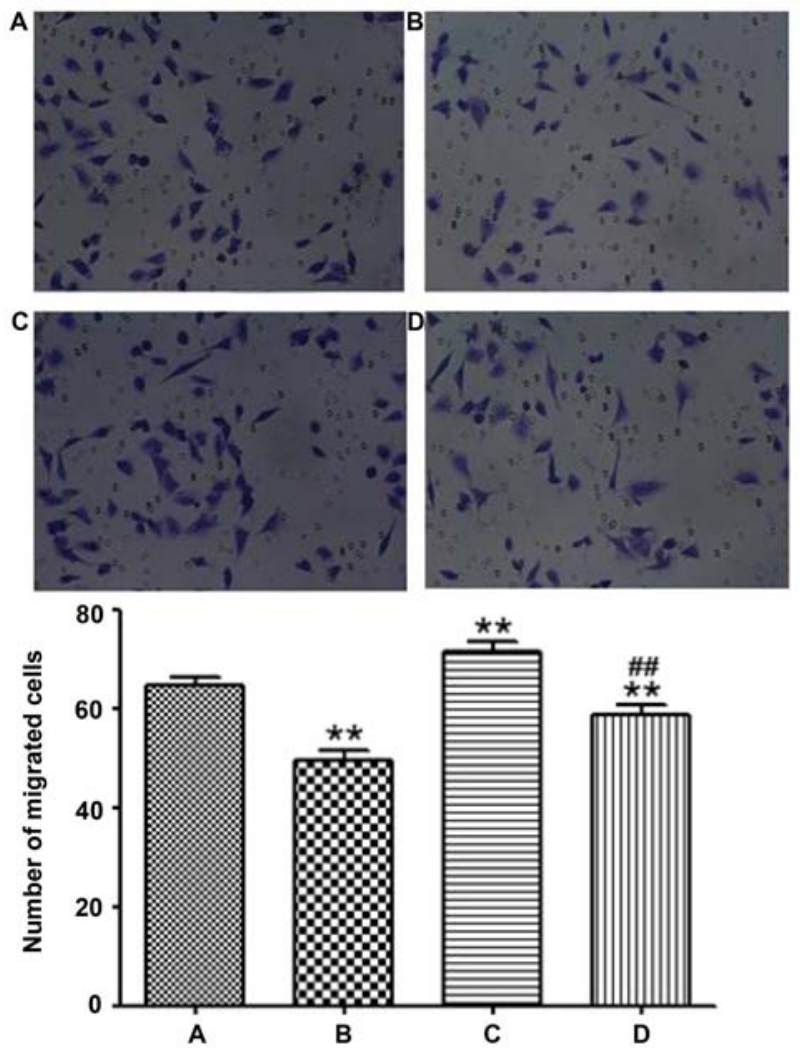

Figure 6. Migration of CRC cells in the different groups (magnification, $\mathrm{x} 200$ ). (A) Blank control group. (B) Metformin group. (C) LKB1 siRNA group. (D) LKB1 siRNA+metformin group. ${ }^{* *} \mathrm{P}<0.01 \mathrm{vs}$. blank control group, ${ }^{\# \#} \mathrm{P}<0.01$ ( $\mathrm{p}-\mathrm{LKB} 1$ ) vs. LKB1 siRNA group. LKB1, liver kinase B1 gene; p, phosphorylated; CRC, colorectal cancer.

capacities were decreased in the metformin group compared with the blank control group. The migration and invasion capacities were decreased in the LKB1 siRNA+metformin group compared with the LKB1 siRNA group. The results of the present study suggest that metformin may be associated with the inhibition of migration and invasion-associated protein expression in LoVo cells.

Effects of metformin on the expression of LKBland p-LKB1 in LoVo cells. To confirm the effect of metformin on the
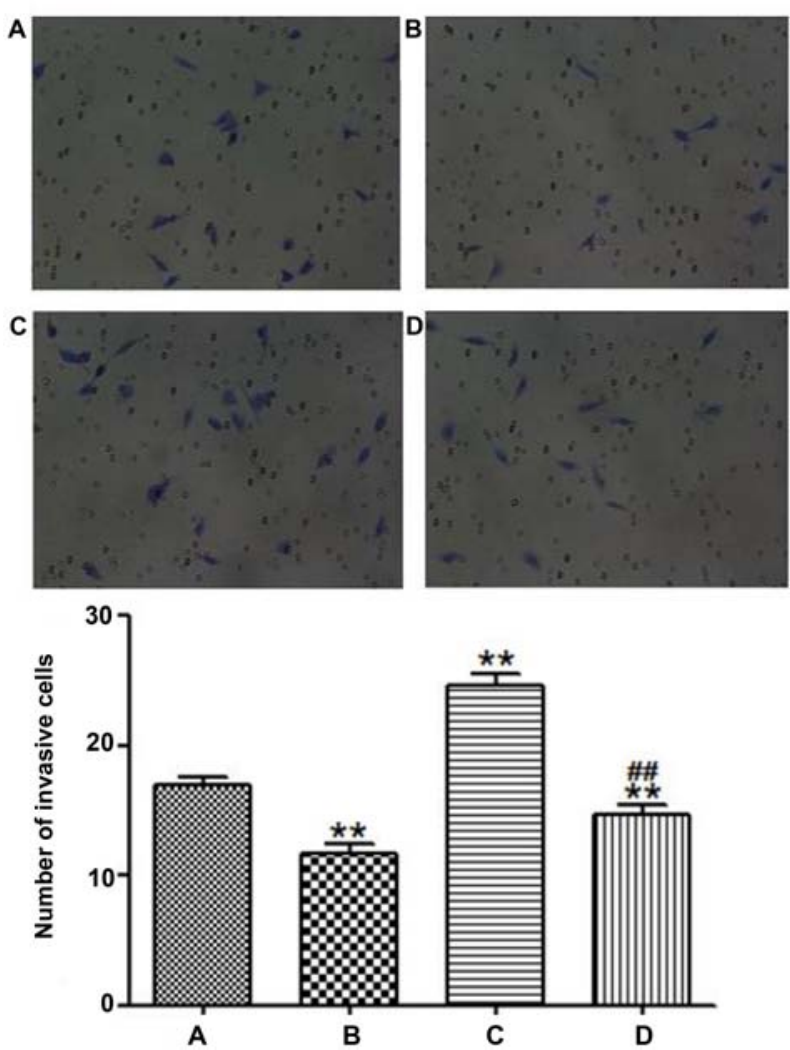

Figure 7. Invasion of CRC cells in the different groups (magnification, $\mathrm{x} 200$ ). (A) Blank control group. (B) Metformin group. (C) LKB1 siRNA group. (D) LKB1 siRNA+metformin group. ${ }^{* *} \mathrm{P}<0.01$ vs. blank control group, ${ }^{\# \#} \mathrm{P}<0.01$ (p-LKB1) vs. LKB1 siRNA group. LKB1, liver kinase B1 gene; p, phosphorylated; CRC, colorectal cancer.

expression of LKB1 and p-LKB1 in LoVo cells, western blot analysis was performed. The protein expression levels of LKB1, p-LKB1 and p-LKB1/LKB1 were significantly reduced in the LKB1 siRNA+metformin group compared with the blank control group $(\mathrm{P}<0.01)$. The protein expression levels of LKB1, p-LKB1 and p-LKB1/LKB1 were significantly increased in the LKB1 siRNA+metformin group compared with the LKB1 siRNA group $(\mathrm{P}<0.01$; Fig. 8). 

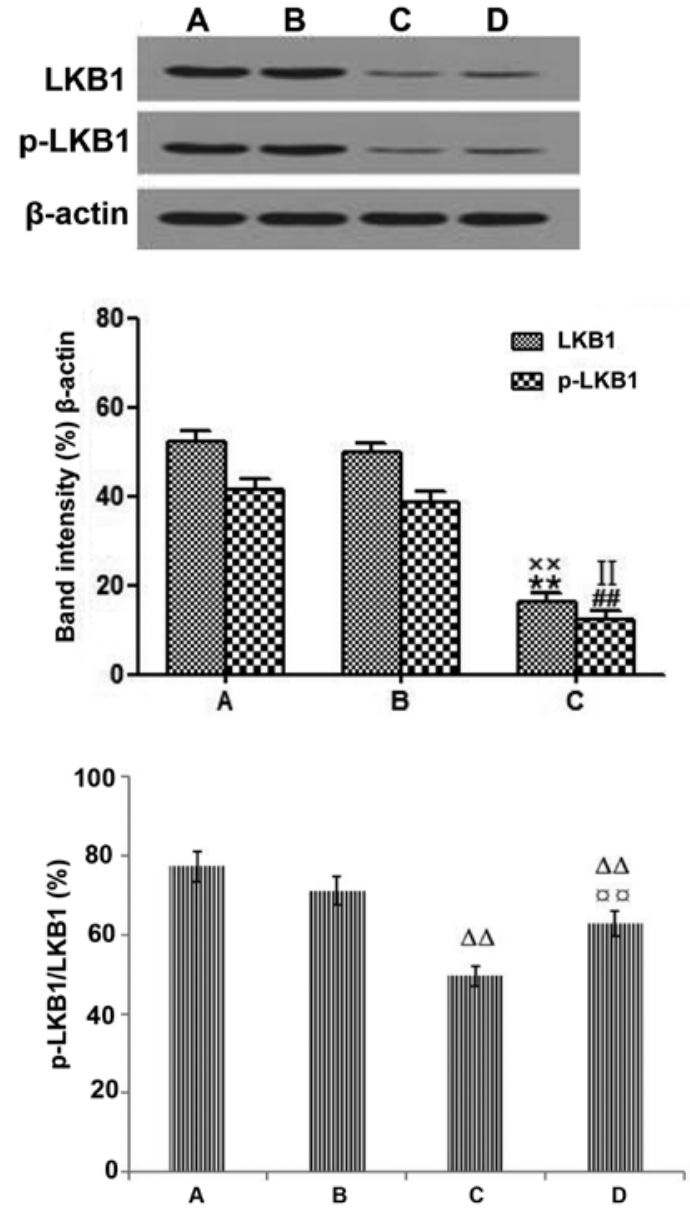

Figure 8. Effects of metformin on the expression of LKB1 and p-LKB1 in CRC LoVo cells. Western blot analysis showed the expression levels of LKB1 and p-LKB1 in LoVo cells. (A) Blank control group. (B) Metformin group. (C) LKB1 siRNA group. (D) LKB1 siRNA+metformin group. ${ }^{* *} \mathrm{P}<0.01$ (LKB1) vs. blank control group, ${ }^{\# \#} \mathrm{P}<0.01$ (p-LKB1) vs. blank control group ${ }^{\mathrm{xx}} \mathrm{P}<0.01$ (LKB1) vs. LKB1 siRNA group, ${ }^{\mathrm{II}} \mathrm{P}<0.01$ (p-LKB1) vs. LKB1 siRNA group. ${ }^{\Delta \wedge} \mathrm{P}<0.01$ (p-LKB1/LKB1) vs. blank control group; ${ }^{\circ \mathrm{a}} \mathrm{P}<0.01$ (p-LKB1/LKB1) vs. LKB1 siRNA group. LKB1, liver kinase B1 gene; p, phosphorylated; CRC, colorectal cancer.

Effects of metformin on the expression of AMPK and p-AMPK in LoVo cells. Western blot analysis showed that the expression of AMPK was not significantly different among the 4 groups $(\mathrm{P}>0.05)$. The expression level of $\mathrm{p}$-AMPK was significantly increased in the metformin group compared with the blank control group $(\mathrm{P}<0.01)$. The expression of p-AMPK and p-AMPK/AMPK were significantly decreased in the LKB1 siRNA group compared with the blank control group $(\mathrm{P}<0.01)$. However, the expression level of $\mathrm{p}-\mathrm{AMPK}$ and p-AMPK/AMPK in the LKB1 siRNA+metformin group were much higher than that in the blank control group, suggesting that the expression of p-AMPK may play a role in CRC. Treatment with metformin increased the protein expression of p-AMPK compared with that of the LKB1 siRNA group, but it did not increase the expression of AMPK in the LKB1 siRNA+metformin group, which indicated that the effect of metformin on the p-AMPK protein expression was dependent on LKB1 ( $\mathrm{P}<0.01$; Fig. 9). These results suggest that further investigation of the biological functions of the activation of AMPK in CRC is needed.
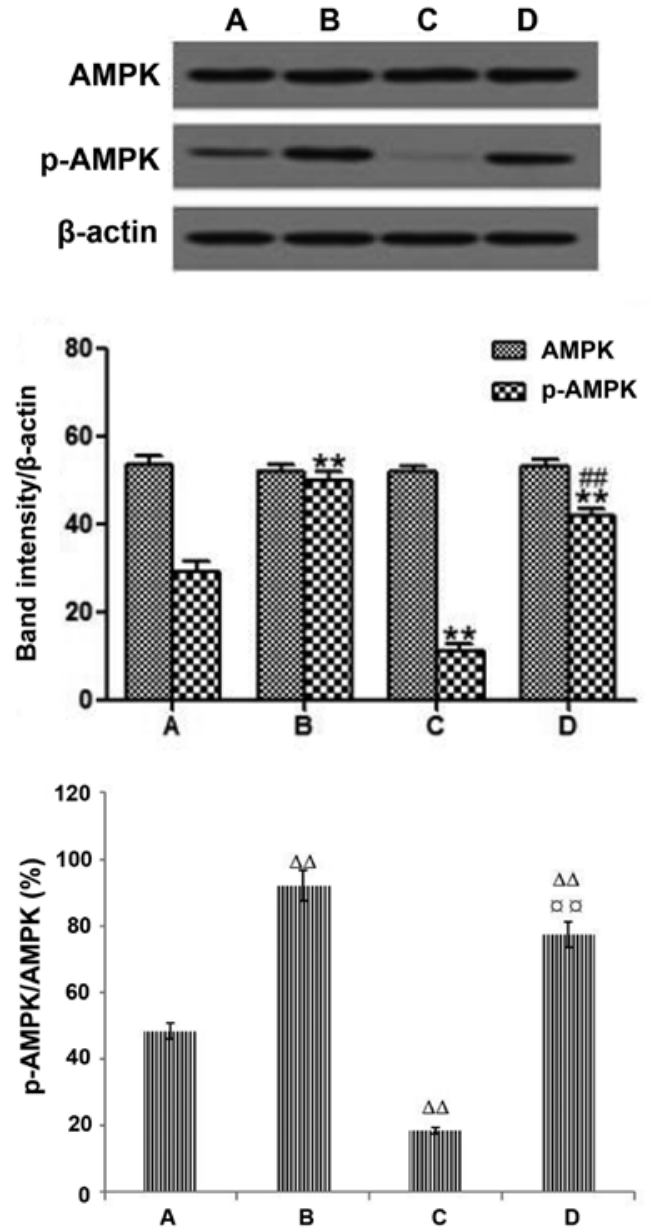

Figure 9. Effects of metformin on the expression of AMPK and p-AMPK in CRC LoVo cells. Western blot analysis showed the expression levels of AMPK and p-AMPK in LoVo cells. (A) Blank control group. (B) Metformin group. (C) LKB1 siRNA group. (D) LKB1 siRNA+metformin group. ${ }^{* *} \mathrm{P}<0.01$ (AMPK) vs. blank control group, ${ }^{\# \#} \mathrm{P}<0.01$ (p-AMPK) vs. LKB1 siRNA group. AMPK, AMP-activated protein kinase. ${ }^{\Delta \Delta} \mathrm{P}<0.01$ (p-AMPK/AMPK) vs. blank control group; ${ }^{a d} \mathrm{P}<0.01$ (p-AMPK/AMPK) vs. LKB1 siRNA group. LKB1, liver kinase B1 gene; AMPK, AMP-activated protein kinase; p, phosphorylated; CRC, colorectal cancer.

Effects of metformin on the expression of AKT and p-AKT in LoVo cells. Western blot analysis showed that the expression of AKT was not significantly different among the 4 groups $(\mathrm{P}>0.05)$. Treatment with metformin decreased the protein expression of $\mathrm{p}$-AKT compared with that of the blank control group. The LKB1 siRNA group had increased protein expression of $\mathrm{p}-\mathrm{AKT}$. The protein expression of $\mathrm{p}-\mathrm{AKT}$ and p-AKT/AKT were reduced in the LKB1 siRNA+metformin group compared with the LKB1 siRNA group. These results indicated that metformin has an effect on p-AKT protein expression and suggested that the AKT signaling pathway may be associated with the metformin-induced changes in the expression of migration and invasion-associated proteins in LoVo cells (Fig. 10).

\section{Discussion}

LKB1 gene expression is closely related to the development and metastasis of colorectal cancer (CRC). By detecting the expression of LKB1 in CRC, we can provide a reference index 

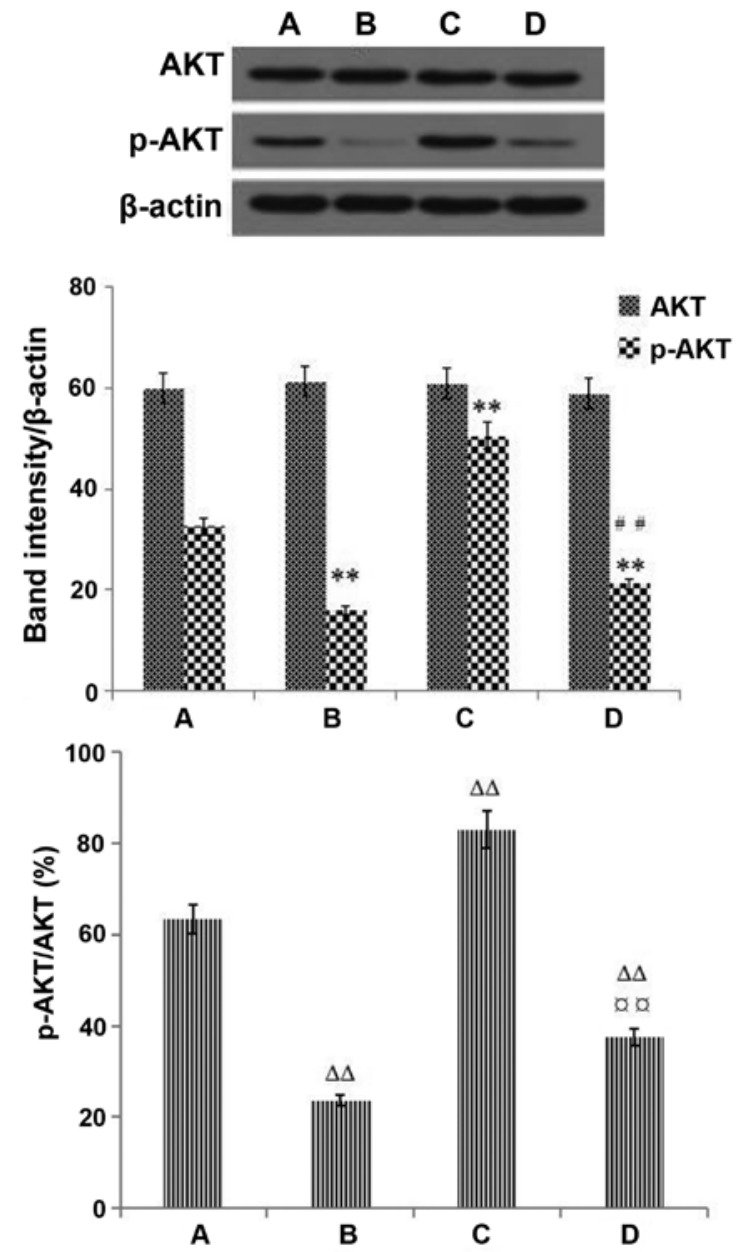

Figure 10. Effects of metformin on the expression of AKT and p-AKT in CRC LoVo cells. Western blot analysis showed the expression levels of AKT and p-AKT in LoVo cells. (A) Blank control group. (B) Metformin group. (C) LKB1 siRNA group. (D) LKB1 siRNA+metformin group. ${ }^{* *} \mathrm{P}<0.01$ (AKT) vs. blank control group, ${ }^{\# \#} \mathrm{P}<0.01$ (p-AKT) vs. LKB1 siRNA group. ${ }^{\Delta \Delta} \mathrm{P}<0.01$ (p-AKT/AKT) vs. blank control group; ${ }^{\text {ax }} \mathrm{P}<0.01$ (p-AKT/AKT) vs. LKB1 siRNA group. LKB1, liver kinase B1 gene; AKT, protein kinase B; p, phosphorylated; CRC, colorectal cancer.

for diagnosing the depth of invasion and lymph node metastasis. It has been reported that the mutation rate of LKB1 in sporadic left colon cancer is $52.6 \%$ (26). In the present study, IHC results showed that expression of LKB1 as a tumor-suppressor gene was strongly positive in normal colorectal tissue, indicating that it can inhibit the occurrence of CRC. The expression of LKB1 was reduced in inflammatory polyp tissue. However, the expression of LKB1 was significantly decreased in adenocarcinoma and mucinous adenocarcinoma tissues, inducing the invasion and metastasis of CRC. These results provide a reference for further investigation of the role of LKB1 in the invasion and metastasis of CRC cells from the perspective of cytology.

New methods based on siRNA can effectively reduce tumor cell volume and inhibit tumor cell repair. siRNA technology has brought new possibilities for gene therapy for CRC patients (13). In the present study, LKB1 siRNA was transfected into LoVo cells to observe the effect of LKB1 on the invasion and metastasis of CRC and the potential underlying mechanism of that effect. As an important tumor-suppressor gene, LKB1 plays a key regulatory role in the LKB1/AMPK/AKT signaling pathway. The results of the western blot analysis showed that the deletion of LKB1 led to a decrease in p-AMPK in the downstream pathway, which promoted $\mathrm{p}-\mathrm{AKT}$, tumor cell proliferation and angiogenesis and enhanced the migration and invasion of CRC cells. The present study used CRC cells to observe the effect of LKB1 on invasion and metastasis of CRC cells. This in vitro experiment had limitations. Further reseach will be carried out by us in in vivo experiments to observe the effect of LKB1 on CRC metastasis in nude mice.

Studies have shown that metformin-induced AMPK activation can reduce protein synthesis and cell proliferation in CRC $(27,28)$. Metformin induces cell cycle arrest or apoptosis, activates the immune system, improves immunity and eliminates cancer stem cells. Increasing numbers of clinical studies have shown that metformin as a typical adjuvant chemotherapy can reduce the risk of invasion and metastasis in cancer patients, resulting in more successful chemotherapy (29). Therefore, we used metformin treatment to observe the effect of metformin on the motility and invasiveness of CRC cells to investigate the possible mechanism by which LKB1 acts on the AMPK-AKT signaling pathway in CRC metastasis. Metformin-induced AMPK activation reduced protein synthesis and cell proliferation and inhibited p-AKT production in CRC cells, thereby inhibiting tumor metastasis, promoting tumor cell apoptosis and decreasing tumor cell migration and invasion. Interestingly, our experiments showed that metformin did not directly activate the phosphorylation of LKB1 (p-LKB1). Metformin inhibited the invasion and metastasis of CRC by activating p-AMPK, thereby inhibiting the activation of p-AKT.

In conclusion, our findings demonstrates that LKB1 plays an important role in the invasion and metastasis of CRC by activating AMPK, negatively regulating the AKT signaling pathway and regulating gene expression, which may be a new anti-angiogenesis and antimetastasis target in CRC treatment. Mutation or deletion of LKB1 repair is expected to be a new therapeutic target or clinical biomarker for the treatment of the invasion and metastasis of CRC.

\section{Acknowledgements}

Not applicable.

\section{Funding}

The present study was supported by grants from the Scientific Research Staring Foundation for Returned Overseas Chinese Scholars, the Ministry of Education of China [grant no. (2013) 1792], the Hubei Province Health and Family Planning Commission Research Projects (grant no. WJ2015MB236), and the Xianning Municipal Science and Technology Research and Development Project [grant no. (2013) 28].

\section{Availability of data and materials}

All data generated or analyzed during the present study are included in this published article. 


\section{Authors' contributions}

YZ designed the experiment, analyzed and explained the data, modified the manuscript, and approved the final version of the manuscript. YC performed the cell experiments and data analysis and interpretation and drafted the manuscripts. YL conceived the experiments, obtained, compiled and analyzed experimental data, participated in the drafting of the manuscripts and approved the final manuscript. HY performed the immunohistochemistry experiments and carried out the data analysis. All authors read and approved the manuscript and agree to be accountable for all aspects of the research in ensuring that the accuracy or integrity of any part of the work are appropriately investigated and resolved.

\section{Ethics approval and consent to participate}

The present study was approved by the Ethics Committee of the Hubei University of Science and Technology (Xianning, China). The committee's reference no. is 201601001. The colon cancer tissue microarray was purchased from Fanpu Biotech, Inc. The company ensured ethical approval from the patients, and patient consent for publication; Clinical Research Protocol (version no. 2016LC001) and informed consent (version no. 2016TYS001).

\section{Patient consent to publication}

Not applicable.

\section{Competing interests}

The authors declare that they have no competing interests.

\section{References}

1. Zhang M, Miao F, Huang R, Liu W, Zhao Y, Jiao T, Lu Y, Wu F, Wang X, Wang $\mathrm{H}$, et al: RHBDD1 promotes colorectal cancer metastasis through the Wnt signaling pathway and its downstream target ZEB1. J Exp Clin Cancer Res 37: 22, 2018.

2. Siegel RL, Miller KD and Jemal A: Cancer Statistics, 2017. CA Cancer J Clin 67: 7-30, 2017.

3. Hawley SA, Pan DA, Mustard KJ, Ross L, Bain J, Edelman AM, Frenguelli BG and Hardie DG: Calmodulin-dependent protein kinase kinase-beta is an alternative upstream kinase for AMP-activated protein kinase. Cell Metab 2: 9-19, 2005.

4. Dorward HS, Du A, Bruhn MA, Wrin J, Pei JV, Evdokiou A, Price TJ, Yool AJ and Hardingham JE: Pharmacological blockade of aquaporin-1 water channel by AqB013 restricts migration and invasiveness of colon cancer cells and prevents endothelial tube formation in vitro. J Exp Clin Cancer Res 35: 36, 2016.

5. Slattery ML, Herrick JS, Mullany LE, Samowitz WS, Sevens JR, Sakoda L and Wolff RK: The co-regulatory networks of tumor suppressor genes, oncogenes, and miRNAs in colorectal cancer. Genes Chromosomes Cancer 56: 769-787, 2017.

6. Eshghifar N, Farrokhi N, Naji T and Zali M: Tumor suppressor genes in familial adenomatous polyposis. Gastroenterol Hepatol Bed Bench 10: 3-13, 2017.

7. Tiainen M, Vaahtomeri K, Ylikorkala A and Mäkelä TP: Growth arrest by the LKB1 tumor suppressor: Induction of $\mathrm{p} 21^{\mathrm{WAF} / \mathrm{CIP} 1}$. Hum Mol Genet 11: 1497-1504, 2002.

8. Ylikorkala A, Rossi DJ, Korsisaari N, Luukko K, Alitalo K, Henkemeyer M and Mäkelä TP: Vascular abnormalities and deregulation of $V E G F$ in $L k b 1$-deficient mice. Science 293: 1323-1326, 2001.
9. Hardie DG and Alessi DR: LKB1 and AMPK and the cancer-metabolism link-ten years after. BMC Biol 11: 36, 2013.

10. Jeon SM and Hay N: The double-edged sword of AMPK signaling in cancer and its therapeutic implications. Arch Pharm Res 38: 346-357, 2015.

11. Larsen AK, de Gramont A, Poindessous V, Bouygues A, Ayadi M and Mésange P: Functions and clinical implications of autocrine VEGF signaling in colorectal cancer. Curr Colorect Cancer Rep 9: 270-277, 2013.

12. Deguchi A, Miyoshi H, Kojima Y, Okawa K, Aoki M and Taketo MM: LKB1 suppresses p21-activated kinase-1 (PAK1) by phosphorylation of $\mathrm{Thr}^{109}$ in the p21-binding domain. J Biol Chem 285: 18283-18290, 2010.

13. Kim SW: The role of MicroRNAs in colorectal cancer. Korean J Gastroenterol 69: 206-211, 2017 (In Korean).

14. You HX, Zhou YH, Tan SY and She TH: Effects of silencing RIP1 with siRNA on the biological behavior of the LoVo human colon cancer cell line. Oncol Lett 7: 2065-2072, 2014.

15. He G, Zou L, Zhou L, Gao P, Qian X and Cui J: Cysteine-rich intestinal protein 1 silencing inhibits migration and invasion in human colorectal cancer. Cell Physiol Biochem 44: 897-906, 2017.

16. Zakikhani M, Dowling RJ, Sonenberg N and Pollak MN: The effects of adiponectin and metformin on prostate and colon neoplasia involve activation of AMP-activated protein kinase. Cancer Prev Res 1: 369-375, 2008.

17. Song X, Kim SY, Zhang L, Tang D, Bartlett DL, Kwon YT and Lee YJ: Role of AMP-activated protein kinase in cross-talk between apoptosis and autophagy in human colon cancer. Cell Death Dis 5: e1504, 2014.

18. Lipovka Y and Konhilas JP: AMP-activated protein kinase signalling in cancer and cardiac hypertrophy. Cardiovasc Pharm Open Access 4: 154, 2015.

19. Xu H, Zhou Y, Coughlan KA, Ding Y, Wang S, Wu Y, Song P and Zou MH: AMPKa1 deficiency promotes cellular proliferation and DNA damage via p21 reduction in mouse embryonic fibroblasts. Biochim Biophys Acta 1853: 65-73, 2015.

20. Su KH, Yu YB, Hou HH, Zhao JF, Kou YR, Cheng LC, Shyue SK and Lee TS: AMP-activated protein kinase mediates erythropoietin-induced activation of endothelial nitric oxide synthase. J Cell Physiol 227: 3053-3062, 2012.

21. Monteverde T, Muthalagu N, Port J and Murphy DJ: Evidence of cancer-promoting roles for AMPK and related kinases. FEBS J 282: 4658-4671, 2015.

22. Wang Y, Wang B, Guerram M, Sun L, Shi W, Tian C, Zhu X, Jiang $Z$ and Zhang L: Deoxypodophyllotoxin suppresses tumor vasculature in HUVECs by promoting cytoskeleton remodeling through LKB1-AMPK dependent Rho A activation. Oncotarget 6: 29497-29512, 2015.

23. Altomare DA and Testa JR: Perturbations of the AKT signaling pathway in human cancer. Oncogene 24: 7455-7464, 2005.

24. Brugarolas J and Kaelin WG Jr: Dysregulation of HIF and VEGF is a unifying feature of the familial hamartoma syndromes. Cancer Cell 6: 7-10, 2004.

25. Amin MB, Edge SB, Greene FL, Byrd DR, Brookland RK, Washington MK, Gershenwald JE, Compton CC, Hess KR, Sullivan DC, et al: AJCC Cancer Staging Manual, 8th edition. 2018.

26. Dong SM, Kim KM, Kim SY, Shin MS, Na EY, Lee SH, Park WS, Yoo NJ, Jang JJ, Yoon CY, et al: Frequent somatic mutations in serine/threonine kinase 11/Peutz-Jeghers syndrome gene in left-sided colon cancer. Cancer Res 58: 3787-3790, 1998.

27. Li W, Wang QL, Liu X, Dong SH, Li HX, Li CY, Guo LS, Gao JM, Berger NA, Li L, et al: Combined use of vitamin D3 and metformin exhibits synergistic chemopreventive effects on colorectal neoplasia in rats and mice. Cancer Prev Res 8: 139-148, 2015.

28. Zaafar DK, Zaitone SA and Moustafa YM: Role of metformin in suppressing 1,2-dimethylhydrazine-induced colon cancer in diabetic and non-diabetic mice: Effect on tumor angiogenesis and cell proliferation. PLoS One 9: e100562, 2014.

29. Wu CY, Wang CJ, Tseng CC, Chen HP, Wu MS, Lin JT, Inoue H and Chen GH: Helicobacter pylori promote gastric cancer cells invasion through NF-kappaB and COX-2-mediated pathway. World J Gastroenterol 11: 3197-3203, 2005. 